\title{
ANÁLISIS FISH EN Pisum sativum Y P. fulvum CON SECUENCIA TELOMÉRICA DE ARABIDOPSIS Y RIBOSÓMICA DE SOYA ${ }^{1}$
}

\author{
Alfredo Bolaños-Herrera ${ }^{2}$, Adoración Cabrera-Caballero ${ }^{3}$, Rocío Recio-Castro ${ }^{4}$
}

\section{RESUMEN}

Análisis FISH en Pisum sativum y $P$. fulvum con secuencia telomérica de arabidopsis y ribosómica de soya. La secuencia 18/25S rDNA de la soja y la TTTAGGG de $A$. thaliana fueron utilizadas como sondas con el objetivo de analizar la organización de los genomas de Pisum sativum, $P$. fulvum y las líneas $\mathrm{F}_{4}$ (P. sativum $\mathrm{X} P$. fulvum) con la técnica de la hibridación in situ fluorescente (FISH). El experimento se realizó en la Universidad de Córdoba, España, en el verano del 2006. La sonda 18/25S produjo señales de intensidad variable en las tres líneas. Estas señales corresponden con los pares de organizadores nucleolares (NOR) localizados en los cromosomas 4 y 7 de $P$. sativum y 4,7 y 5 de $P$. fulvum. La intensidad de las señales varió de muy fuerte a medianamente fuerte en $P$. sativum, a pequeñas y discretas en $P$. fulvum, lo que sugiere que existen diferencias en el número de veces que la secuencia se repite en los genomas de estas dos especies. Las señales que se observaron en la línea $\mathrm{F}_{4}$ con la sonda de rDNA se asemejaron en tamaño e intensidad a las que se observaron en P. sativum y P. fulvum. Las muestras de las líneas $\mathrm{F}_{4}$ evaluadas presentaron dos NOR, si bien en una de ellas se observó una señal muy baja en un tercer par de cromosomas. La secuencia TTTAGGG hibridó en los telómeros de las tres líneas estudiadas, lo que mostró que la secuencia telomérica de A. thaliana está también presente en el género Pisum.

Palabras clave: Citogenética, in situ fluorescente, NOR, 18/25S rDNA, TTTAGGG.

\begin{abstract}
FISH analysis in Pisum sativum and $P$. fulvum with a telomeric sequence from Arabidopsis and a ribosomic one from soybean. The sequence $18 / 25 \mathrm{~S}$ rDNA from soybean and the TTTAGGG from A. thaliana were used as probes to analyze the genomic organization of Pisum sativum, $P$. fulvum and a F4 line (P. sativum X P. fulvum) by fluorescent in situ hybridization (FISH). The experiment was conducted at the University of Córdoba, Spain during the summer of 2006. The probe $18 / 25 \mathrm{~S}$ produced variable signals in the three genotypes. These rDNA signals correspond to the nucleolar organizing pairs (NOR`s) on chromosomes 4 and 7 of $P$. sativum, and 4, 7 and 5 of P. fulvum. The intensity of signals varied from very strong to moderately-strong in $P$. sativum to small and secrete in $P$. fulvum, suggesting clear differences on the number of times that the sequence $18 / 25 \mathrm{~S}$ is repeated in the genome of these two species. Signals observed in the F4 line using the rDNA probe resembled $P$. sativum and $P$. fulvum knobs. Two NOR's were observed on the F4 line, although one of the lines showed very low signals for one pair of chromosomes. The TTTAGGG sequence hybridized with the telomeres of the three lines, which shows that the telomeric sequence from A. thaliana is also present in the Pisum genera.
\end{abstract}

Key words: Cyto-genetics, fluorescent in situ hybridization, NOR, 18/25S rDNA, TTTAGGG.

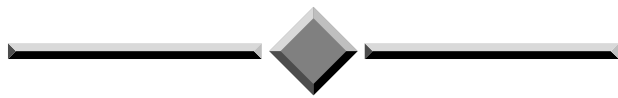

Recibido: 21 de diciembre, 2007. Aceptado: 19 de marzo, 2009. Parte de la tesis doctoral del primer autor.

2 Instituto Nacional de Innovación y Transferencia en Tecnología Agropecuaria (INTA), Costa Rica. Sabana Sur, San José, Costa Rica. ab24@cornell.edu

3 Departamento de Genética y Mejora Vegetal, Escuela Técnica Superior de Ingenieros Agrónomos y Montes (ETSIAM). Universidad de Córdoba, edificio Gregor J. Mendel Campus de Rabanales, 14071 Córdoba España. ge1cabca@uco.es; rore7@hotmail.com 


\section{INTRODUCCIÓN}

La hibridación interespecífica es una estrategia para la mejora vegetal, que tiene como objetivo ampliar la base genética de las variedades cultivadas y proporcionar los genes necesarios para reducir o anular el efecto negativo, de las plagas y los componentes abióticos del ambiente, sobre el rendimiento y calidad de las cosechas. La arveja o guisante (Pisum sativum L.) $(2 n=2 x=14)$ no escapa a las consideraciones anteriores (Fuchs et al. 1998, Tar'an et al. 2003, Tar'an et al. 2005). Dentro del género Pisium, especial interés ha recibido la especie silvestre $P$. fulvum $(2 \mathrm{n}=2 \mathrm{x}=14)$, en la que se han identificado genes de resistencia a varias plagas (Fondevilla 2005, Hardie et al. 1995, Sharma y Yadav 2003). Aún cuando existe cierto nivel de incompatibilidad entre estas dos especies (Ochatt et al. 2004) originada posiblemente en diferencias morfológicas entre cromosomas homeólogos o en la distribución de secuencias repetidas en el genoma (Schwarzacher 2003) varios genes de resistencia a plagas han sido introducidos con éxito en $P$. sativum (Fondevilla 2005, Fondevilla et al. 2005).

La hibridación in situ fluorescente (FISH) es una técnica robusta que permite identificar cada uno de los cromosomas de una determinada especie (Cerbah et al. 1999, Fuchs et al. 1998, Samatadze et al. 2005, Schneider et al. 2003, Vischi et al. 2003) distinguir en un individuo la localización y magnitud del aporte cromosómico de cada uno de los progenitores (Samatadze et al. 2005, Soliman et al. 2001).

La localización por FISH de secuencias repetidas de la familia de genes nrDNA permite localizar los organizadores nucleolares (NOR) ya que algunas secuencias de esta familia de genes hibridan con señales fuertes en estas zonas (Schmidt et al. 1994, Fuchs et al. 1998, Cerbah et al. 1999, Falistocco et al. 2002, Prieto et al. 2004) y pueden utilizarse como puntos físicos de referencia tanto para la identificación de un brazo de brazos cromosómicos en particular, como para la integración de los mapas de ligamiento con los mapas físicos (Sadder y Weber 2002, Stephens et al. 2004).

Las sondas construidas con secuencias repetidas teloméricas (TTTAGGG) son de utilidad para visualizar la estructura de los cromosomas, éstas hibridan al final de los cromosomas únicamente (Fuchs et al. 1998, Sykorova et al. 2003) aunque esta secuencia no se presenta en todas las plantas (Adams et al. 2000) y en algunos casos secuencias teloméricas heterólogas pueden no hibridar en todos los cromosomas o bien hacerlo con intensidad variable (Pedrosa et al. 2002). Las secuencias subteloméricas o asociadas a los telómeros hibridan en otros sitios de los brazos de los cromosomas (Kolchinsky y Gresshoff 1994) con lo que pueden producir patrones válidos para comparar genomas.

En la presente investigación se utilizó la hibridación in situ fluorescente (FISH) para comparar la organización de los genomas de P. sativum y P. fulvum mediante la utilización de sondas correspondientes a secuencias de rDNA de soja y telomérica de Arabidopsis.

\section{MATERIALES Y MÉTODOS}

Esta investigación se realizó en los laboratorios del Departamento de Genética y Mejora Vegetal de la Escuela Técnica Superior de Ingenieros Agrónomos y Montes (ETSIAM) Universidad de Córdoba, España, durante el verano del 2006.

\section{Material vegetal y preparación de los cromosomas}

Como material vegetal se utilizaron secciones de raíces de aproximadamente $1 \mathrm{~cm}$ de largo, que se obtuvieron a partir de semillas germinadas en cámara húmeda del cultivar Ballet ( $P$. sativum) de $P$. fulvum (línea P660) y de plantas correspondientes al cuarto ciclo de autopolinización $\left(\mathrm{F}_{4}\right)$ del híbrido obtenido de la cruza entre Ballet y P660 (amablemente cedidas por el Dr. Diego Rubiales del Consejo Superior de Investigaciones Científicas (CSIC), Córdoba). Las raíces y preparaciones se procesaron según el protocolo utilizado en el laboratorio para raíces de trigo y otras gramíneas (Cabrera et al. 2002). Las secciones de raíz se colocaron en solución de colchicina al $0,05 \%$ durante tres horas en la oscuridad y luego se colocaron en viales con una solución de etanol:ácido acético 3:1 (v:v) y se guardaron en refrigeración $\left(4^{\circ} \mathrm{C}\right)$ hasta la preparación de las muestras cromosómicas. Para la preparación de las muestras, se sumergió una raíz en carmín acético por uno o dos minutos, tras lo cual se colocó sobre un vidrio porta objetos donde se cortó transversalmente una sección muy fina, la que se humedeció con una gota de ácido acético al 45\%. De seguido se cubrió con un vidrio cubre objetos sobre el que se colocó un papel y se aplicó presión con el dedo pulgar. Finalmente se pasó la preparación sobre la llama de un mechero para 
luego localizar los cromosomas en un microscopio de fase.

Las preparaciones en las que se encontraron metafases somáticas con buena morfología, se congelaron en nitrógeno líquido, se les removió el cubre objetos y luego de que se secaron a $23^{\circ} \mathrm{C}$ y $60 \% \mathrm{HR}$ (RT) se guardaron en refrigeración hasta realizar el análisis FISH.

\section{Sondas, preparación y marcaje}

La secuencia completa 18/25 rDNA aislada de la soja se marcó con biotina (Roche 1093070) por Nick Translation siguiendo el protocolo del fabricante (Kit de Boehringer 976776). La detección se hizo con rodamina. La secuencia telomérica aislada de Arabidopsis thaliana pAtT4 (Richards y Ausbel 1988) se marcó también por Nick Translation con dioxigenina (Roche 1109088910) siguiendo el protocolo del fabricante (Kit de Boehringer 976776). La detección de la sonda se realizó con fluoresceína.

\section{Hibridación in situ fluorescente y localización de las sondas en las preparaciones cromosómicas}

El análisis FISH se realizó según el protocolo utilizado en el laboratorio para muestras de trigo y otros cereales (Cabrera et al. 2002). Las preparaciones se incubaron por dos horas a $37^{\circ} \mathrm{C}$ con 50 ul de solución de RNAsa (100 ul 20XSSC, 10 ul RNAsa y 890 ul de agua estéril) cubiertas con un cubre objetos. Seguidamente se lavaron dos veces con solución 2XSSC por cinco minutos a temperatura ambiente (RT) y una vez a $37^{\circ} \mathrm{C}$ por cinco minutos. Luego se trataron en una solución al $1 \%$ de pepsina (al $5 \%$ ) y $1 \% \mathrm{HCl} 1 \mathrm{M}$ en agua destilada por 10 minutos a $37^{\circ} \mathrm{C}$. A continuación se lavaron dos veces en buffer salino de fosfato (PBS 1X) por cinco minutos a RT. De seguido se trataron con una solución al $1 \%$ formaledehido en PBS $10 \mathrm{X}, 50 \mathrm{mM}$ ClMg por 10 minutos a RT. Por último se volvieron a lavar en PBS $1 X$ por cinco minutos a RT. Antes de aplicar las sondas, las preparaciones se deshidrataron con un lavado en etanol al $75 \%$ y otro con etanol al $100 \%$ por tres minutos cada uno y se dejaron secar a $23{ }^{\circ} \mathrm{C}$ y $60 \% \mathrm{HR}$ (RT) sobre la mesa del laboratorio.

Una vez secas las preparaciones, cada una se trató con $2 \mathrm{ng} / \mathrm{ul}$ de cada sonda disueltas y desnaturalizadas en la mezcla de hibridación, que consistió de una solución al 50\% de formamida desionizada y 10\% de sulfato de dextrano y $10 \%$ de 2 XSSC en agua destilada.
Para la desnaturalización de las sondas, la mezcla se calentó a $75{ }^{\circ} \mathrm{C}$ durante ocho minutos tras lo cual se colocó en hielo picado por cinco minutos. Las preparaciones se cubrieron con cubre objetos y se procedió a la desnaturalización conjunta de los cromosomas en las preparaciones y las sondas aplicadas. Para ello se colocaron las muestras en un termociclador programado en la siguiente secuencia: siete minutos a $75^{\circ} \mathrm{C}$, dos minutos a $55^{\circ} \mathrm{C}, 30$ segundos a $50^{\circ} \mathrm{C}$, un minuto a 45 ${ }^{\circ} \mathrm{C}$, dos minutos a $42{ }^{\circ} \mathrm{C}$, cinco minutos a $40{ }^{\circ} \mathrm{C}$ y cinco minutos a $38{ }^{\circ} \mathrm{C}$. Seguidamente las preparaciones se encubaron en cámara húmeda a $37^{\circ} \mathrm{C}$ por 16 horas.

Tras la incubación, las preparaciones se lavaron dos veces con una solución de $2 \mathrm{XSSC}$ a $37{ }^{\circ} \mathrm{C}$ por cinco minutos y dos veces más con la misma solución a media concentración (1SSC) por cinco minutos a RT. Seguidamente se trataron con una solución de Tris- $\mathrm{HCl} 0,1 \mathrm{M}, \mathrm{NaCl} 0,15 \mathrm{M}$ y 0,05\% Tween-20 en agua destilada (TNT) por cinco minutos a RT seguida por el tratamiento con una solución de Tris- $\mathrm{HCl} 0,1 \mathrm{M}$, $\mathrm{NaCl} 0,15 \mathrm{M}$ y $0,05 \%$ Blocking Reagent (Boerhinger) en agua destilada (TNB) a $37^{\circ} \mathrm{C}$ por 20 minutos. A continuación se lavaron con una solución al $0,05 \%$ de tween-20 en 4XSSC (4T). Seguidamente se aplicaron 35 ul de solución de TNB con los anticuerpos estreptavidina y antidigoxigenina con una concentración de 10 ng/ul de cada uno y se encubaron en cámara húmeda por 45 minutos en la oscuridad. Seguidamente se lavaron en TNT por cinco minutos en la oscuridad a RT y se deshidrataron con un lavado en etanol $70 \%$ seguido de otro en etanol al $100 \%$ para dejarlos secar al aire en oscuridad y a RT. Seguidamente las preparaciones se contratiñeron con 4',6-Diamidino-2-fenil indol diclorhidrato (DAPI, Sigma D9542), 35 ul por preparación, se cubrieron con cubre objetos y se encubaron por cinco minutos en cámara húmeda en la oscuridad y RT. Por último se lavaron por cinco minutos con agitación en agua estéril en oscuridad y a RT, seguido de la cual se deshidrataron en etanol en la misma secuencia que las veces anteriores, pero únicamente por un minuto. Las preparaciones se dejaron secar en la oscuridad y se almacenaron en refrigeración a $4{ }^{\circ} \mathrm{C}$ hasta su análisis en el microscopio.

\section{Procesado de las imágenes fotográficas}

La detección de las sondas se realizó con un microscopio LEICA DMR usando los filtros apropiados. Las imágenes se tomaron con una cámara SPOT Jr 
CCD. Las imágenes se procesaron con el programa de cómputo Spot Jr (Diagnostics Instruments, Inc.).

\section{RESULTADOS}

\section{Sonda de rDNA}

La sonda rDNA produjo señales fuertes, de intensidad similar, en cuatro de los 14 cromosomas de $P$. sativum, mientras que en $P$. fulvum la misma sonda originó seis señales, dos de intensidad similar a las observadas en $P$. sativum y dos de intensidad media (Figura 1). Estos resultados indican que P. sativum presentó dos pares de NOR mientras que $P$. fulvum presentó tres pares. En las muestras de las plantas $\mathrm{F}_{4}$, la sonda hibridó en cuatro sitios del genoma con señales de alta intensidad y en dos sitios con intensidad muy baja (Figura 2). Estas señales corresponderían con los NOR en ambas especies parentales. En una de las muestras $\mathrm{F}_{4}$ que se evaluaron, además de las cuatro señales de hibridación, se observó una señal intersticial de intensidad muy baja (Figura 2). P. sativum tiene dos NOR (cromosomas 4 y 7) (Fuchs et al. 1998, Samatadze et al. 2002) mientras que $P$. fulvum tiene tres (cromosomas 4, 7 y 5) (Errico et al. 1991, Errico et al. 1996, 1997) de ahí que los cromosomas de $P$ sativum en los que se observaron las señales (Figura $1 \mathrm{~A}, \mathrm{~B}, \mathrm{C}$ ) corresponderían a los pares 4 y 7 (Fuchs $e t$ al. 1998, Samatadze et al. 2002) y en P. fulvum serían los pares 4, 7 y 5. La intensidad de las señales FISH se relaciona con el número de veces que la secuencia se repite en cada sitio (Sadder y Weber 2002).

Las imágenes obtenidas muestran con claridad que los NOR en $P$. sativum se localizan próximos a los telómeros, mientras que en $P$. fulvum, al menos uno de ellos esta en una zona intersticial de uno de los cromosomas (Figura 1).

Las señales de la sonda de rDNA en algunos de los NOR de P. sativum (Figura 1A y B1) y de P. fulvum (Figura 1D) mostraron un halo de hibridación débil, lo que indica la desconcensación de esa región cromosómica.

\section{La Sonda Telomérica}

La superposición de las imágenes obtenidas con las sondas de rDNA y la sonda telomérica generó señales naranja, que concuerdan con los NOR en ambas especies y en las plantas $F_{4}$, lo que se debe a que debido a la alta intensidad de las señales producidas por la sonda marcada con biotina, éstas son captadas por el microscopio aún cuando se utilice el filtro para visualizar las señales de las sondas marcadas con digoxigenina (Figuras 2C y 2F) igual situación ha sido reportada por Kolchinsky y Gresshhoff (1994). La sonda telomérica hibridó en las regiones distales de los cromosomas de ambas especies de guisante, pero tanto la frecuencia como la intensidad fueron mayores en las preparaciones de P. fulvum (Figura 3). Tampoco

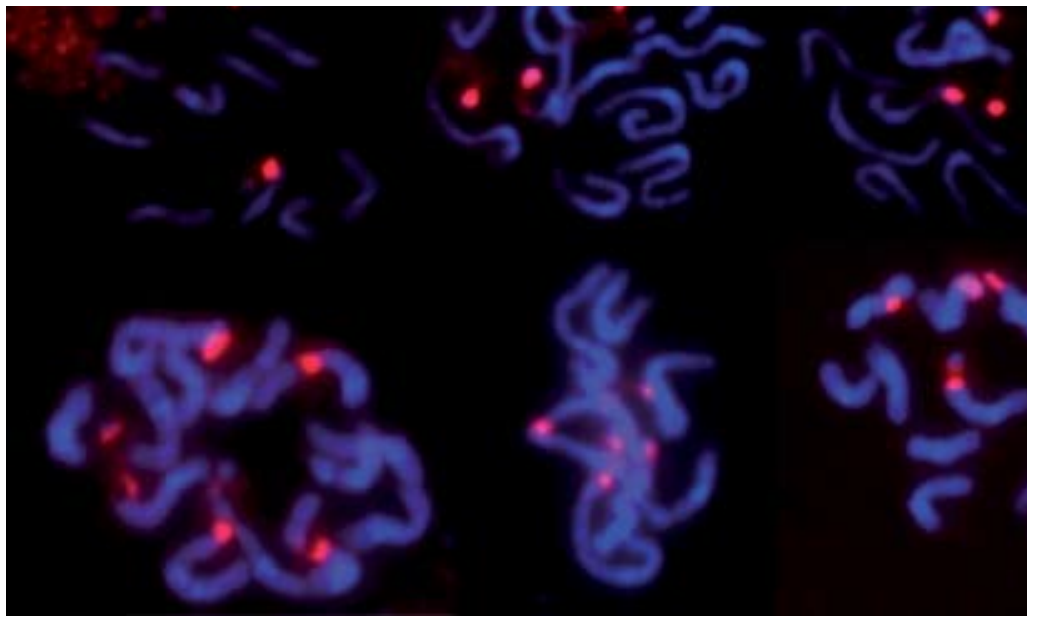

Figura 1. Metafase somática de P. sativum (A, B, C) y P. fulvum (D, E, F) hibridada con la sonda ribosómica marcados con biotina y detectados con rodamina. Los cromosomas se contratiñeron con DAPI. Escuela Técnica Superior de Ingenieros Agrónomos y Montes (ETSIAM). Universidad de Córdoba, España. 2006. 


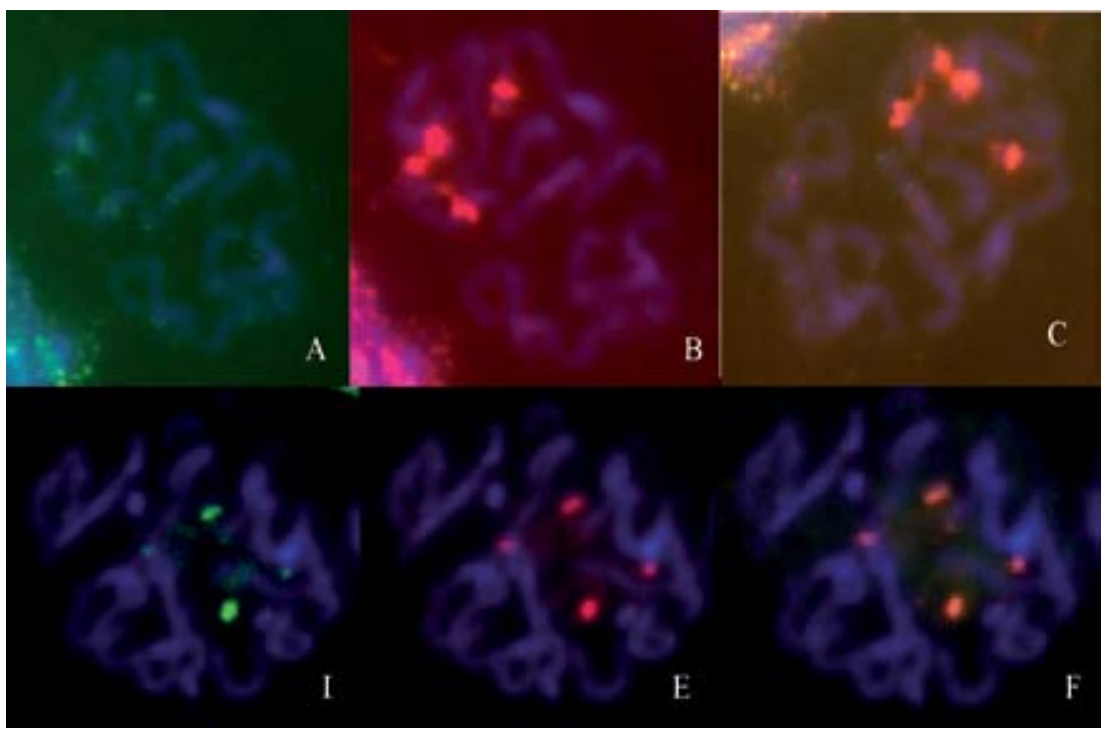

Figura 2. Metafases somáticas de dos plantas $\mathrm{F}_{4}$, hibridadas con la sonda telomérica, detectadas con fluoresceina (verde) (A y D) y con la ribosómica, marcados con biotina y detectados con rodamina (By E) y la superposición de ambas imágenes (C y F). Los cromosomas se contratiñeron con DAPI. Escuela Técnica Superior de Ingenieros Agrónomos y Montes (ETSIAM). Universidad de Córdoba, España. 2006.

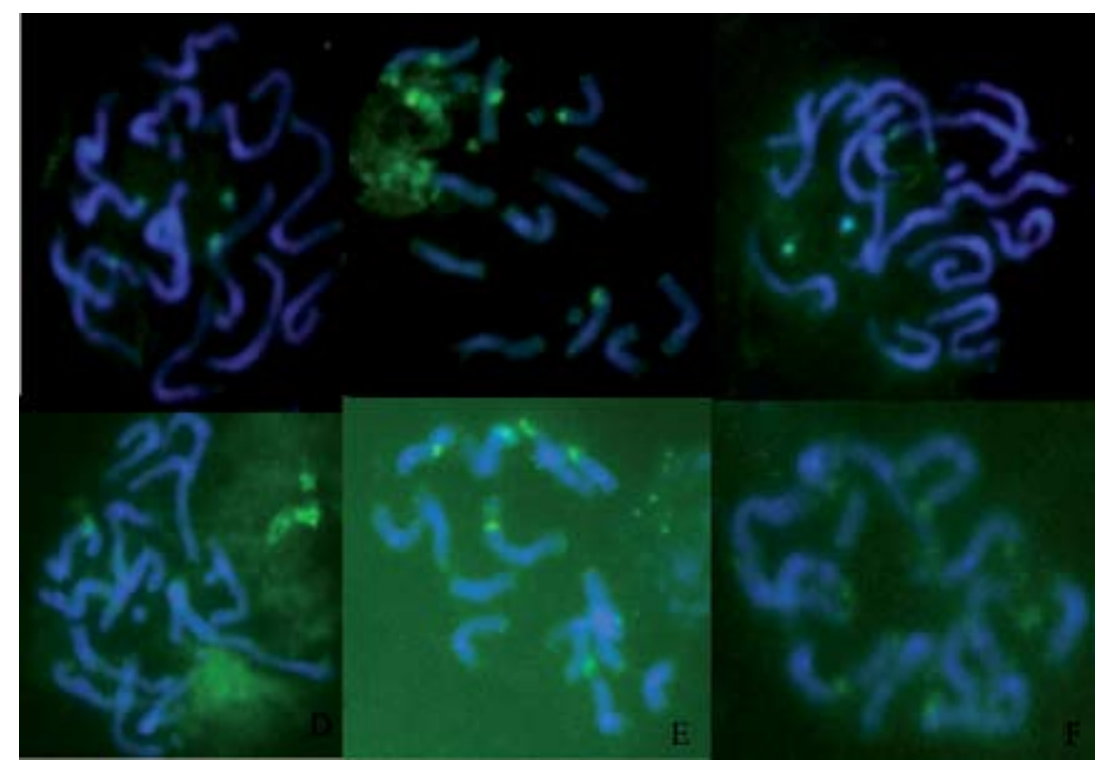

Figura 3. Metafases somáticas de P. sativum (A, B, C) y P. fulvum (D, E, F) hibridadas con la sonda telomérica, detectadas con fluoresceina (verde)con la sonda Telomérica y contra teñidos con DAPI. Escuela Técnica Superior de Ingenieros Agrónomos y Montes (ETSIAM). Universidad de Córdoba, España. 2006. 
las señales fueron visibles con igual intensidad en todas las muestras ni en todos los cromosomas de una preparación, lo que concuerda con lo expuesto sobre este tema por Kolchinsky y Gresshoff (1994) quienes indican que secuencias subteloméricas y otras asociadas a los telómeros, son muy variables en el número de veces que se repiten y aún en cuanto a su ocurrencia en individuos de la misma especie. Este resultado indica que, al igual que otras muchas especies vegetales, los telómeros de $P$. sativum y $P$. fulvum comparten esta secuencia con Arabidopsis thaliana.

\section{DISCUSIÓN}

La hibridación fluorescente in situ de preparaciones cromosómicas de Pisum sativum y P. fulvum con sondas de rDNA y con la secuencia telomérica de $A$. thaliana, permitió establecer diferencias en la organización del genoma de ambas especies. Los resultados mostraron con claridad que ambas especies difieren en el número de NOR y que generaciones avanzadas, obtenidas por auto polinización y originadas del cruzamiento de ambas especies, tienen dos pares de NOR, número característica de $P$. sativum junto con una señal intersticial muy baja en un tercer par de cromosomas. También los datos permiten indicar que mientras que los NOR de $P$. sativum se localizan en la región distal de los cromosomas, en P. fulvum uno de ellos está localizado en la región meridional de un brazo en uno de los cromosomas.

La hibridación con la sonda telomérica de $A$. thaliana, en las regiones distales de los cromosomas de las muestras de Pisum analizadas, muestra que en los telómeros de este género también se encuentra la secuencia TTTAGGG. La presencia de esta secuencia en otra especies vegetales está bien documentada (Fuchs et al. 1998, Prieto et al. 2004, Sykorova et al. 2003) no así para el caso de P. fulvum o P. sativum.

Queda por evaluar un mayor número de líneas producto del cruzamiento entre ambas especies y ampliar el número de sondas, para comprender mejor las diferencias y semejanzas entre el genoma del guisante cultivado, su pariente silvestre y el de las progenies que se obtienen de su hibridación. Estos datos permitirían referenciar los mapas de ligamiento del género con posiciones físicas en los cromosomas, información que será de gran utilidad para la mejora genética de las especies cultivadas del género Pisum.

\section{RECONOCIMIENTOS}

Este trabajo fue parcialmente apoyado por: Plan de Doctores Sistema INIAs-Ibero América, Instituto Nacional de Innovación y Transferencia en Tecnología Agropecuaria (INTA) Costa Rica, Consejo Nacional de Investigaciones Científicas y Tecnológicas (CONICIT) Costa Rica, El Ministerio de Ciencia y Tecnología de Costa Rica (MICIT) y la Universidad de Córdoba, España.

El material vegetal fue suministrado por el Dr. Diego Rubiales, CSIC, Córdoba.

\section{LITERATURA CITADA}

Adams, SP; Leitch, IJ; Bennett, MD; Leitch, AR. 2000. Aloe L. -a second plant family without (TTTAGGG) telomeres. Chromosoma 109: 201-205.

Cabrera, A; Martin, A; Barro, F. 2002. In situ comparative mapping (ISCM) of Glu-1 loci in Triticum and Hordeum. Chromosome Research 10 (1): 49-54.

Cerbah, M; Kevel, Z; Siljak-Yakovlev, S; Kkondorosi, E; Kondorosi, A. 1999. FISH Chromosome mapping allowing karyotype analysis in Mendicago truncatula lines Jemalong J5 and R-108-1. Molecular Plant-Microbe Interations 12 (11): 947-950.

Errico, A; Conicella, C; Taliercio, U. 1991. Cytological and morphological characterization of Pisum sativum and Pisum fulvum tetraploids. Plant Breeding 106 (2): 141-148.

Errico, A; Conicella, C; Martino, TD; Ercolano, R; Monti, LM. 1996. Chromosome reconstructions in Pisum sativum through interspecific hybridization with $P$. fulvum. Journal of Genetics and Breeding 50 (4): 309-313.

Errico, A; Conicella, C; Martino, TD; Ercolano, R; Monti, LM. 1997. Chromosome reconstructions in Pisum sativum through interspecific hybridization with $\mathrm{P}$. fulvum. Journal of Heredity 50 (4): 309-313.

Falistocco, E; Torricelli, R; Falcinelli, M. 2002. Genomic relationships between Medicago murex Willd. and Medicago lesinsii E. Small. investigated by in situ hybridization. Theoretical and Applied Genetics 105 (6-7): 829-833. 
Fondevilla, S. 2005. Mejora del guisante para resistencia a jopo (Orobanche crenata), ascoquitosis (Mycosphaerella pinodes) y oidio (Erysiphe pisi) mediante mejora clásica y marcadores moleculares. Tesis Doctor. Córdoba, España. Universidad de Córdoba. 163 p.

Fondevilla, S; Avila, CM; Cubero, JI; Rubiales, D. 2005. Response to Mycosphaerella pinodes in a germplasm collection of Pisum spp. Plant Breeding 124 (3): 313315.

Fuchs, J; Kühne, M; Schubert, I. 1998. Assignment of linkage groups to pea chromosomes after karyotyping and gene mapping by fluorescent in situ hybridization. Chromosoma 107 (4): 272-276.

Hardie, DC; Baker, GJ; Marshall, DR. 1995. Field screening of Pisum accessions to evaluate their susceptibility to the pea weevil (Coleoptera: Bruchidae). Euphytica. 84 (2): 155-161.

Kolchinsky, A; Gresshoff, P. 1994. Plant telomeres as molecular markers. In: Gresshoff, P. ed. Plant Genome Analysis. Boca Raton, US, CRC. p 113-124.

Ochatt, SJ; Benabdelmouna, A; Marget, P; Aubert, G; Moussy, F; Pontecaille, C; Jacas, L. 2004. Overcoming hybridization barriers between pea and some of its wild relatives. Euphytica 137: 353-359 p.

Pedrosa, A; Sandal, N; Stougaard, J; Schweizer, D; Bachmair, A. 2002. Chromosomal map of the model legume Lotus japonicus. Genetics 161 (4): 1661-1672.

Prieto, P; Martín, A; Cabrera, A. 2004. Chromosomal distribution of telomeric and telomeric-associated sequences in Hordeum chilense by in situ hybridization. Hereditas 141: 1-4.

Richards, EJ; Ausubel, FM. 1988. Isolation of a higher eukaryotic telomere from Arabidopsis thaliana. Cell 53 (1): 127-136.

Sadder, MT; Weber, G. 2002. Comparison between genetic and physical maps in Zea mays L. of molecular markers linked to resistance against Diatraea spp. Theoretical and Applied Genetics 104 (6 - 7): 908-915.

Samatadze, TE; Muravenko, OM; Bol'sheva, NL; Amosova, AB; Gostimscki, SA; Zelenin, AV. 2005. Investigation of

ISSN: 1021-7444 chromosomes in varieties and translocation lines of pea Pisum sativum L. by FISH, Ag-NOR, and differential DAPI staining. Genetika 41 (12): 1665-1673.

Samatadze, TE; Muravenko, OV; Zelenin, AV; Gostimskii, SA. 2002. Identification of the pea (Pisum sativum L.) genome chromosomes using C-banding analysis. Doklady Biological Sciences 387 (1-6): 577-580.

Schmidt, T; Schwarzacher, T; Heslop-harrison, JS. 1994. Physical mapping of rRNA genes by fluorescent in situ hybridization and structural analysis of rRNA genes and intergenic sequences in sugar beet (Beta vulgaris). Theoretical and Applied Genetics 88: 629-636.

Schneider, A; Linc, G; Molnar-Lang, M. 2003. Fluorescence in situ hybridization polymorphism using two repetitive DNA clones in different cultivars of wheat. Plant Breeding 122 (5): 396-400.

Schwarzacher, T. 2003. Meiosis recombination and chromosomes: a review of gene isolation and fluorescent in situ hybridization data in plants. Journal of Experimental Botany 54 (380): 11-23.

Sharma, B; Yadav, Y. 2003. Pisum fulvum carries a recessive gene for powdery mildew resistance. Pisum Genetics 35: sp.

Soliman, MH; Rubiales, D; Cabrera, A. 2001. A fertile amphiploid between durum wheat (Triticum turgidum) and the $\mathrm{x}$ Agroticum amphiploid (Agropyron cristatum x T. tauschii). Hereditas 135 (2-3): 183-186.

Stephens, JL; Brown, RN; Lapitan, NLV; Knudson, DL. D. L. 2004. Physical mapping of barley genes using an ultrasensitive fluorescence in situ hybridization technique. Genome 47 (1): 179.

Sykorova, E; Lim, KY; Chase, MW; Knapp, S; Leitch, IJ; Leitch, AR; Fajkus, J. 2003. The absence of Arabidopsis-type telomeres in Cestrum and closely related genera Vestia and Sessea (Solanaceae): first evidence from eudicots. The Plant Journal 34 (3): 283-291.

Tar'an, B; Warkentin, T; Somers, DJ; Miranda, D; Vandenberg, A; Blade, S; Woods, S; Bing, D; Xue, A; Dekoeyer, D; Penner, G. 2003. Quantitative trait loci for lodging resistance, plant height and partial resistance to mycosphaerella blight in field pea (Pisum

AGRONOMÍA MESOAMERICANA 20(1): 23-30 2009 
sativum L.). Theoretical and Applied Genetics 107 (8): 1482-1491.

Tar'an, B; Zhang, C; Warkentin, T; Tullu, A; Vandenberg, A. 2005. Genetic diversity among varieties and wild species accessions of pea (Pisum sativum L.) based on molecular makers, and morphological and physiological characters. Genome 48 (2): 257-272.

Vischi, M; Jurman, I; Bianchi, G; Morgante, M. 2003. Karyotype of Norway spruce by multicolor FISH. Theoretical and Applied Genetics 107 (4): 591-597. 